\title{
Enhancement of Degraded Historical Kannada Documents
}

\author{
B Gangamma \\ PES Institute of Technology \\ Bangalore, INDIA
}

\author{
Srikanta Murthy K \\ PES School of Engineering \\ Bangalore, INDIA
}

\begin{abstract}
Historical documents play a vital role in understanding our past and hence need to be preserved. Over the period, these documents tend to possess many variations like stains, strain, ink seepage, dust etc. Image enhancement techniques can be utilized to improve the quality of these images by removing noise and increasing contrast range. The proposed method mainly deals with enhancing the historical document image of palm scripts using gray scale morphological operations with the combination of spatial filters. Morphological opening operation is applied to compensate for non uniform background intensity and suppress bright details smaller than the structural element, while closing suppresses the dark details. The proposed method works well for the images with dark background and low contrast and enhances the image with clear white background.
\end{abstract}

\section{Keywords}

Adaptive Histogram Equalization Degraded Documents, Enhancement, Gray Scale Morphological Operators, Inscriptions.

\section{INTRODUCTION}

In India, it is approximately estimated that more than 5 million of documents/manuscripts are available and uncountable inscriptions, documents are spread across the country in form of stone carving, metal inscriptions, palm leaf manuscripts, clothes and paper scripts. Many organizations are working to find, record, preserve and deliver these to connect India's past with its future, its memory with its aspirations. As these documents are degraded in nature, digitization process alone is not sufficient. Hence, it is very important to develop suitable algorithm to enhance these documents for deciphering. It is observed that from the literature survey, many authors have presented a lot of work in the enhancement of the degraded documents using various methods. The enhancement of the document with ink bleed through using recursive unsupervised classification technique has been proposed by Drira [2006]. The presented method performs recursively K-means algorithm on the degraded image with principal component analysis of the document image. Then cluster values are taken and back projected on the space. The iterative method is used for finding logarithmic histogram and separating background and foreground using K-means algorithm until clear separation of background and foreground of the document was made. B. Gatos et al. [2006] presented an adaptive approach for the binarization and enhancement of degraded documents by combining many algorithms. Proposed method uses low-pass Wiener filter for pre-processing, interpolation of neighboring background intensities for rough estimation of foreground regions and background surface calculation, a thresholding by combining the calculated background surface with the original image while incorporating image up-sampling and finally a postprocessing step in order to improve the quality of text regions and preserve stroke connectivity. Krishna Kishore et al. [2007] used Unsharp masking to enhance the edge detail information in the degraded document.

B. Gatos et al. [2008] proposed method mainly based on the combination of several state-of-the-art binarization methodologies as well as on the efficient incorporation of the edge information of the gray scale source image. An enhancement step based on mathematical morphology operations is also involved in order to produce a high quality result while preserving stroke information. Yahia S et al. [2009] presented an enhanced system for degraded old document. The developed system is able to deal with degradations which occur due to shadows, non-uniform illumination, low contrast and noise. Ferhat et al. [2010] proposed image restoration using Singular Value Decomposition and restored even blurred image.

Even though literature survey reveals research work on Indian languages, the dedicated research is required to address the degraded documents. In this paper, we attempted to enhance, one of the South Indian language, Kannada documents. The proposed method uses simple and efficient combination of spatial filter and morphological operations which are based on set theory concepts as these are computationally efficient with respect to time. Very few authors have used mathematical morphology for document image enhancement. Xiangyun Ye et al. [1999] had proposed elimination of background using morphological operations. Santhosh et al. [2000] has proposed a method for background elimination of bank cheques using gray scale morphological operations. This paper presents a simple and efficient method for elimination of the background and enhances the degraded documents.

\section{PROPOSED METHOD}

This paper presents an efficient method to enhance the degraded documents which comprise of many problems, like low contrast, uneven illumination, and noise. The method uses adaptive histogram equalization; gray scale morphological operators and spatial filters to enhance the low contrast suppress the noise as well as background intensity and enhance the image respectively. 


\subsection{Adaptive Histogram Equalization (AHE)}

The binarization process separates foreground text from background. If the histogram of the image is bimodal or sparsely distributed, then binarization process separates text from background. Degraded historical document as shown in Figure 1(a) poses low contrast and noise accumulation. It will be difficult to separate text from background using simple thresholding technique. The result of thresholding using Otsu method is as shown in Figure 1(b). Histogram equalization will be used in contrast stretching for low contrast images. There are two types of histogram equalization, one global and other local. Global histogram can be used to enhance the contrast of the image by computing single histogram and redistributing the intensities across the image. This works better for low contrast image with fairly uniform background. Adaptive Histogram Equalization (AHE) is used to overcome uneven background and illumination and uses local histograms for each sub image, unlike global histogram equalization.

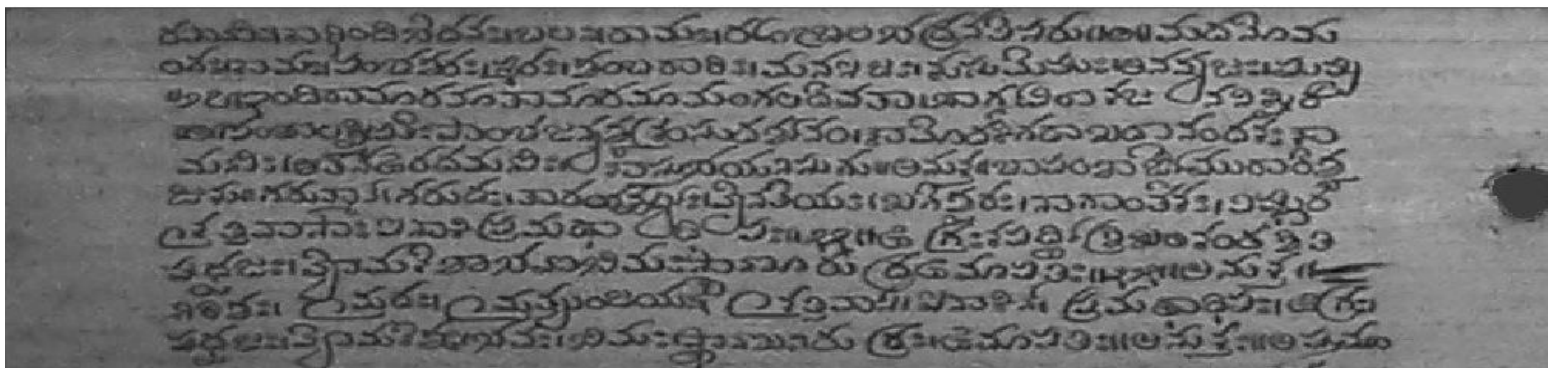

Fig 1 (a) Original Degraded Document

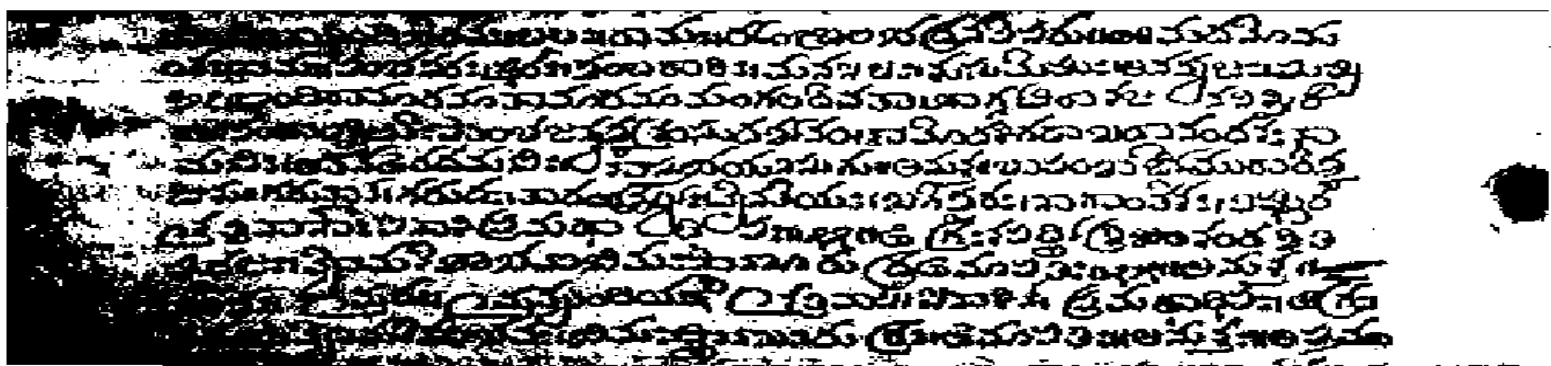

Fig 1 (b) Binarized with Otsu method.

\subsection{Morphology}

Image-processing techniques such as digital multimedia and computer vision have been developed tremendously during the past five decades and among them, mathematical morphology has received a great interest because it provides a quantitative description of geometric structure and shape as well as a mathematical description of algebra, topology, probability, and integral geometry [Frank. Y. Shih 2010].

Mathematical Morphology is a tool used for extracting image components that are useful for representation and description of region of shape, such as boundaries, skeletons and the convex hull. It can also be used as a tool for pre or post processing such as, morphological filtering, thinning, and pruning [Rafael C Gonzalez 2008]. It is a set-theoretic method of image analysis providing a quantitative description of geometrical structures. Most morphological operations are based on simple expanding and shrinking operations. The primary application of morphology occurs in binary images, though it is also used on gray level images. It can also be useful on range images.

The two basic morphological set transformations are erosion and dilation. These transformations involve the interaction between an image $A$ (the object of interest) and a structuring set $B$, called the structuring element. Typically the structuring element $B$ is a circular disc or rectangle in the plane, but it can be of any shape and any dimension.

Dilation: With $\mathrm{A}$ and $\mathrm{B}$ as sets in $\mathrm{Z}^{2}$ (set of Integer), the dilation of A by B denoted as $\boldsymbol{A} \oplus \boldsymbol{B}$, is defined as

$$
A \oplus B=\left\{Z \mid(\hat{B})_{z} \cap \neq \emptyset\right\}
$$

Erosion: With A and B as sets in $\mathrm{Z}^{2}$ (set of Integer), the erosion of $\mathrm{A}$ by $\mathrm{B}$ denoted as $\boldsymbol{A} \ominus \boldsymbol{B}$, is defined as

$$
A \ominus B=\left\{z \mid(B)_{z} \subseteq A\right\}
$$

where $\mathrm{A}$ in equation (1) and (2), is the object image and B is structuring element of any size, but less than or equal to the size of A. Dilation expands the region of the object. Erosion shrinks or thins objects in an image. Figure 2 shows the result of dilation and erosion on A image by structuring element B.

Opening and Closing: Erosion and dilation can be used in a variety of ways, in parallel and series, to give other transformations including thickening, thinning, skeletonization and many others. 


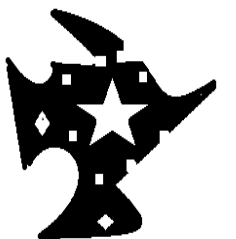

(a)

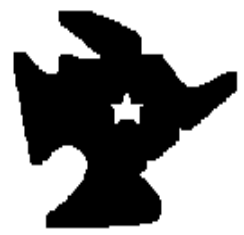

(b)

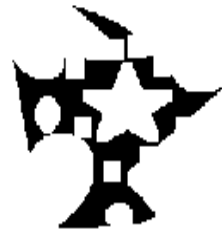

(c)
Fig 2 Binary Morphological Operations on Image (a) with disk structuring element of radius 5. (b) Result of Dilation and (c) Result of Erosion.

Two very important transformations are opening and closing. Opening generally smoothes contour in an image, breaking narrow isthmuses and eliminating thin protrusions. Closing tends to narrow smooth sections of contours, fusing narrow breaks and long thin gulfs, eliminating small holes, and filling gaps in contours. The opening of image $A$ by structuring element $B$, denoted by $A \circ B$, is given by the erosion by $B$, followed by the dilation by $B$, that is

$$
A \circ B=(A \ominus B) \oplus B
$$

Opening is like 'rounding from the inside'. The opening of $A$ by $B$ is obtained by taking the union of all translates of $B$ that fit inside $A$, parts of $A$ that are smaller than $B$ are removed.

Closing is the dual operation of opening and is denoted by $A \bullet B$. It is produced by the dilation of $A$ by $B$, followed by the erosion by $B$ :

$$
A \cdot B=(A \oplus B) \ominus B
$$

Figure (3) shows the application of opening and closing operation and its output. Opening operation smooth the object from the outside. Holes are filled in and narrow valleys are closed in closing operation. Because opening suppresses the bright details smaller than the structuring element and closing suppress dark details, they are used in combination for image smoothing and noise removal. Opening can be used to compensate for non uniform background illumination. Also subtracting opened image from Original image produces even background [Rafael C Gonzalez 2008].

\subsection{Gray Scale Morphology}

In binary morphology both image A and structuring element B are binary, the operations applied on the two sets are logical operations such as AND, OR, and COMPLEMENT, commonly referred as binary morphology. The output is also in the form binary. Gray scale morphology operations uses finding local maxima and minima in specified window. In many cases, grayscale morphological processing adopts symmetrical structuring elements so as to reduce computational complexity. [Frank. Shih 2010]. The erosion of A by structuring element B at any location $(x, y)$ is defined as the minimum value of the image in the specified local region centered at $(x, y)$. Gray scale erosion is defined by following equation

$$
[A \ominus B](x, y)=\min _{(s, t) \in B}\{f(x+s, y+t)\}
$$

Gray scale erosion computes the minimum intensity value of A in every local region, eroded image will be darker than original image. Noise smaller than structuring element will be eliminated. The gray scale dilation of A by B is defined by finding the maximum value of the image in the window outlined by $\mathrm{B}$ and is given by

$$
[A \oplus B](x, y)=\max _{(s, t) \in B}\{f(x-s, y-t)\}
$$

\section{Opening and Closing}

Formulae for opening and closing for gray scale morphology are same as binary morphology as specified in equation (3) and (4).

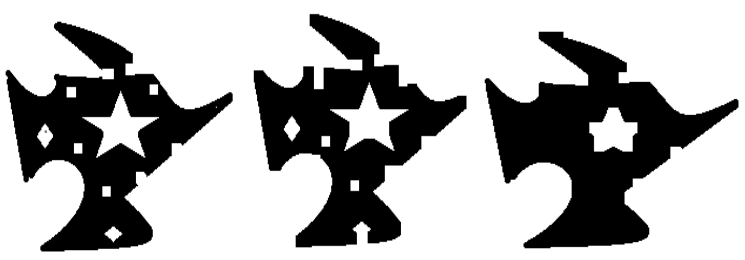

Fig 3 (a)Original Image (b) Result of opening operation on image, (c) Result of closing operation on image.

\section{ALGORITHM}

The proposed method uses simple and efficient spatial domain techniques for contrast enhancement and linear smoothing filter with combination of non linear filters using gray scale morphological operations to eliminate the background and to enhance the degraded image. The degraded noisy original image, shown in Figure 4(a) with uneven illumination is taken as input and AHE is applied to get a histogram equalized image and will be referred further as AHE image. Morphological grayscale opening operation is applied to get opened image. Addition of Figure 4(b) with histogram equalized image produces an image with clear background shown in Figure 4(c). Image in Figure 4(c) is enhanced with good contrast showing clear separation of text and background. Then morphological closing is applied to this image in order to smooth the image and result is shown in Figure 4(d). The AHE image is subtracted from image and result is again subtracted from Figure 4 (c). The reconstructed images are shown in Figure 4 (e-f). Gaussian smoothing filter is applied to eliminate noise to image Figure 4(g). Final enhanced image is reconstructed by adding the to the AHEd image to get enhanced image with almost white background and foreground with characters alone, shown in Figure 4(h). Thresholding or binarization is required to segment the image into text and background, well known global thresholding method by Otsu's [1979], is applied to binarize the enhanced image (Figure 4(i). The proposed method is very fast as it uses simple linear and non linear filter, computational cost is very less and takes approximately 1 second to process 500x3500 pixel size image.

\section{RESULTS AND DISCUSSION}

Experimentation has been done on more than 150 different degraded historical palm documents. Results have shown that the proposed method is well suited for enhancing degraded documents with low contrast and noise. Figure (5) shows experimental results for paper document and Figure (7) shows for palm script image. The proposed method is compared with Average filter, Gaussian filter and adaptive thresholding and results are shown Figure (6). 


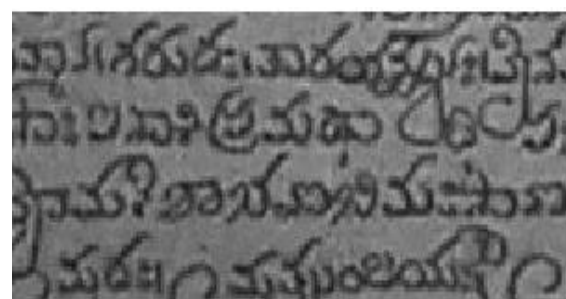

Fig 4. (a) Original Image

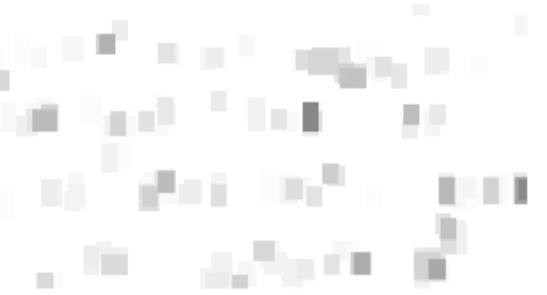

Fig 4. (d) Result of Closing Operation

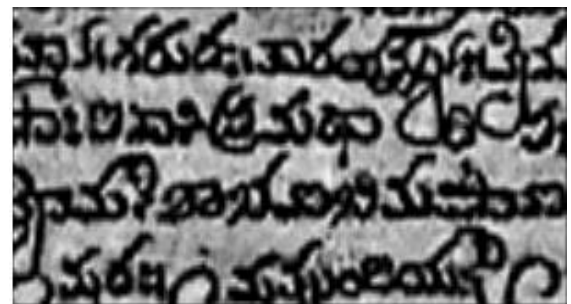

Fig 4. (g) Result of Gaussian filter on image(f)

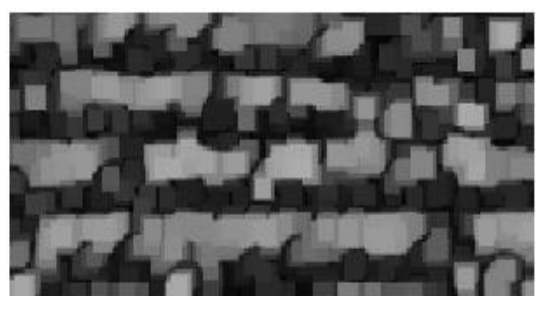

Fig 4. (b) Result of Opening operation on AHE.

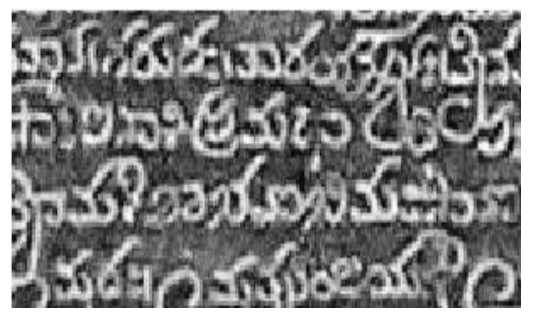

Fig 4. (e) Reconstructed image from of result of closing and Original image.

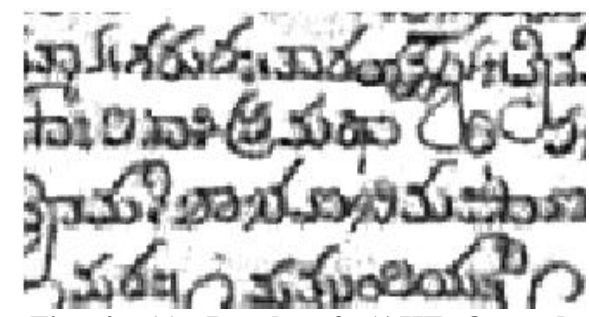

Fig 4. (c) Result of (AHE+Opened image).

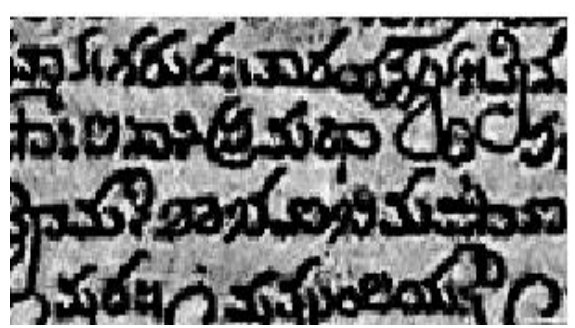

Fig 4. (f) Result of Reconstruction

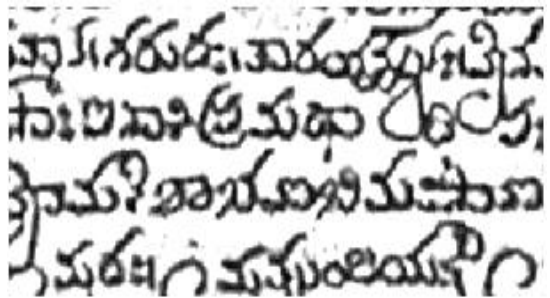

Fig 4. (h) Result of adding image ' $g$ ' and AHE image.

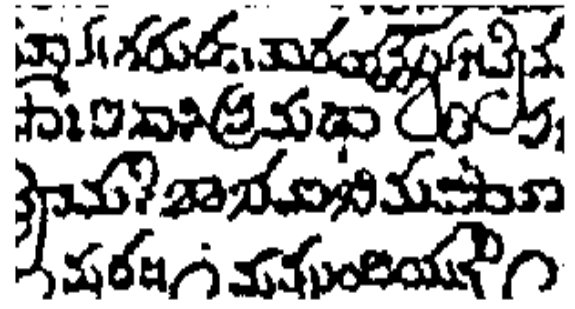

Fig 4. (i)Result of Otsu binarization method.

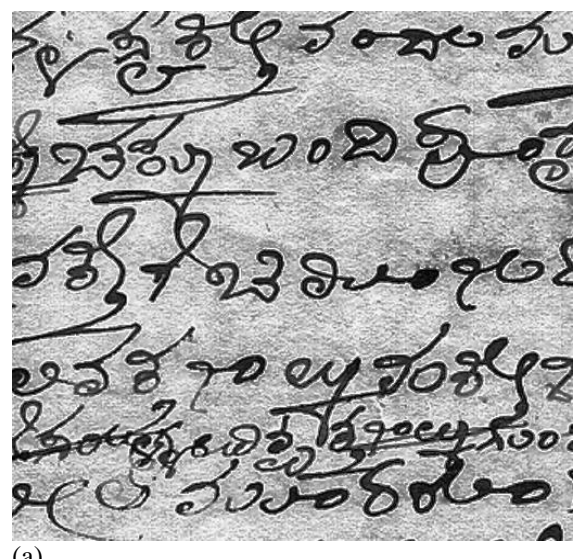

(a)
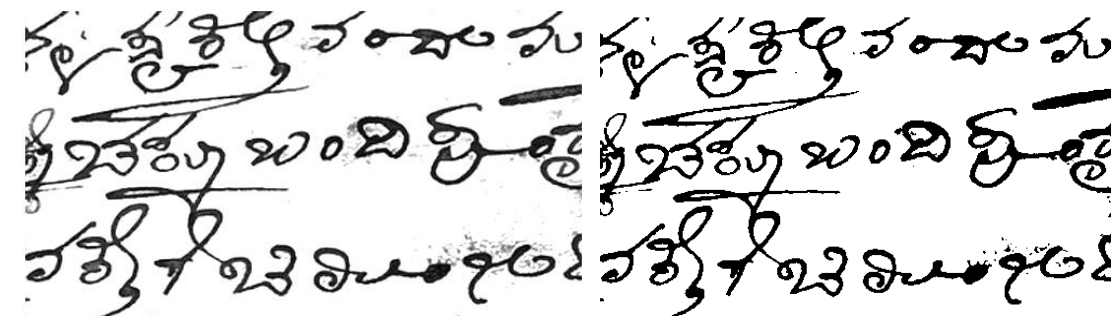
68

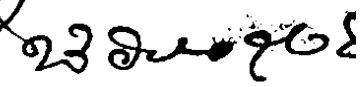

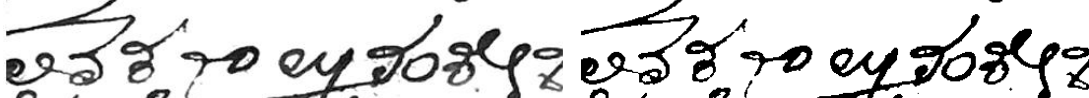

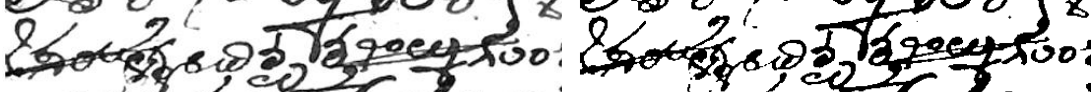
\&CS

(b) (c)

Fig 5 (a) Original Image, (b) Enhanced Image using proposed method. (c) Binarized output image. 
Average and Gaussian filters are well known smoothing algorithm to eliminate the noise. If the filter size is more, the smoothing algorithm produces blurred image and making binarization task very difficult. Usually Palm scripts are dark brown due to application of oil and disinfectant to keep them for longer time. These are usually low contrast with characters submerged with background making smoothing operation difficult. The proposed method enhances the image by eliminating noisy background and produces clear image with almost white background thus making binarization task easier. Any character recognition system is completely depends upon the result of binarization method so that segmentation, feature extraction and classification of the document should be accurate.

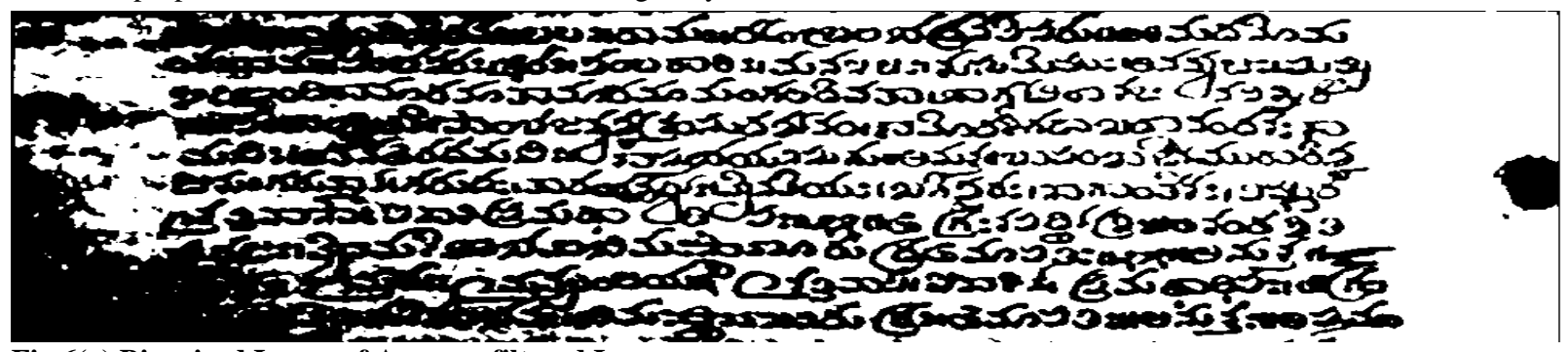

Fig 6(a) Binarized Image of Average filtered Image.

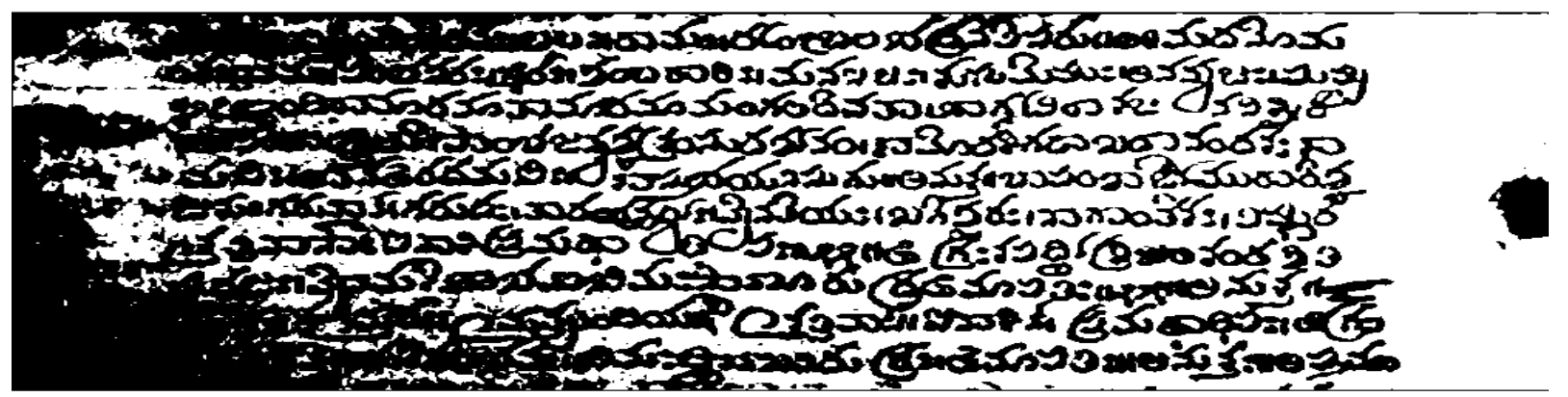

Fig 6(b) Binarized Image of Gaussian filtered Image

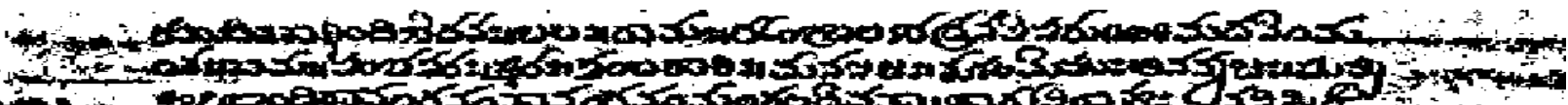

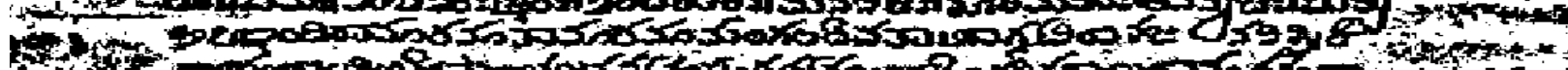

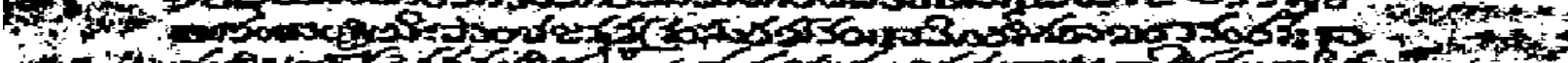

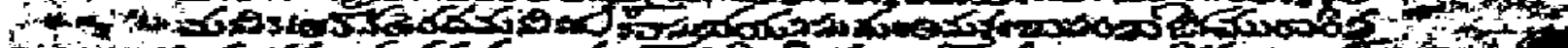

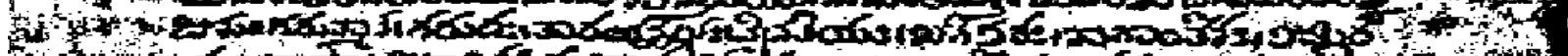

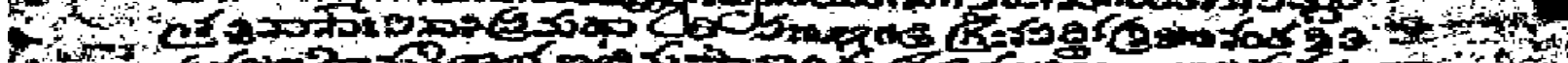

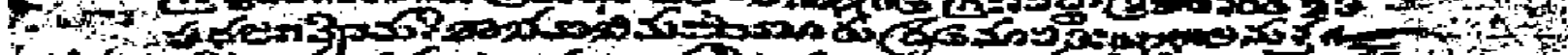

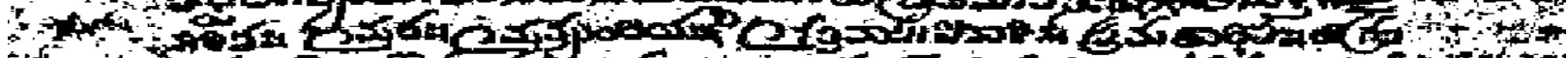
of Fig 6(c) Binarized Image using Âdaptive Thresholding

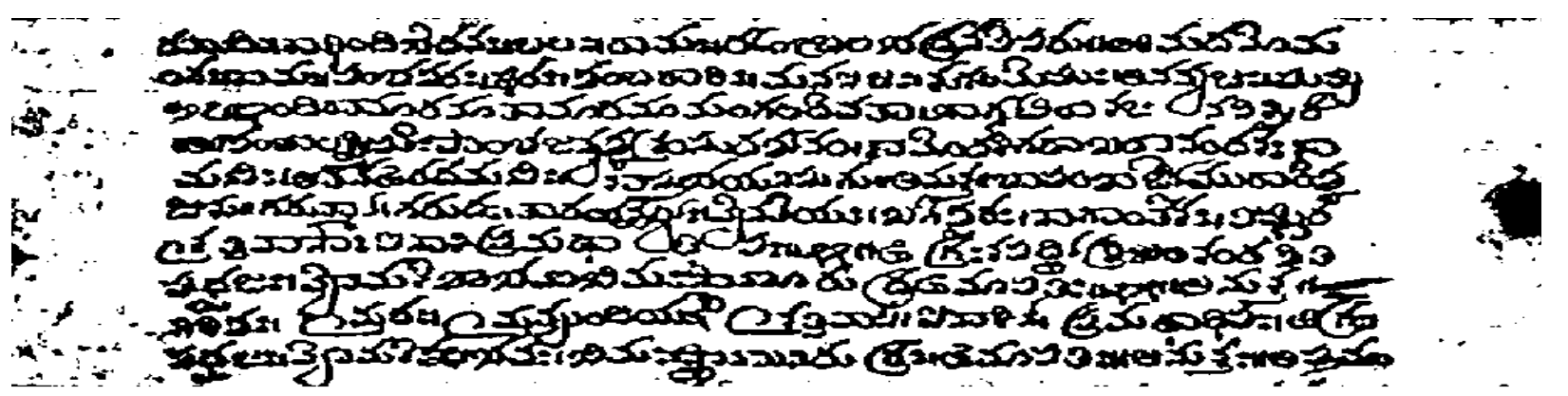

Fig 6 (d) Binarized Image of enhanced Image using Proposed Method. 


\section{CONCLUSION}

Simple and efficient method for enhancement of degraded historical document has been proposed. This method enhances the degraded document image mathematical morphological operations that are developed to quantitatively describe the shape and size of images. The proposed method uses adaptive histogram equalization for contrast enhancement followed by morphological operations as these are simple and powerful tool to eliminate noise, remove background and produce an enhanced image with pure foreground contents. Blurred and skewed images are not in the scope of the present work.

\section{REFERENCES}

[1] Drira Fadoua, Frank Le Bourgeis and Hubert Emptoz[2006], "Restoring Ink Bleed Through Degraded Document Images Using a Recursive Unsupervised Classification Technique". Spinger-Verlag Berlin Heidelberg, DAS LNCS 3872, pp. 38-49.

[2] B. Gatos, I. Pratikakis, S.J. Perantonis[2006], "Adaptive Degraded Document Image Binarization”, Journal of Pattern Recognition 39, 317 - 327.

[3] N. Krishna Kishore, Priti P. Rege[2007], “Adaptive Enhancement of Historical Document Images", IEEE International Symposium on Signal Processing and Information Technology, 978-1 -4244-1 835-0/07, pp 98388.

[4] B. Gatos, I. Pratikakis and S.J. Perantonis[2008], "Improved Document Image Binarization by Using a Combination of Multiple Binarization Techniques and Adapted Edge Information", 19th International Conference on Pattern Recognition (ICPR'08), ISBN: 978-1-42442175-6/08.
[5] Yahia S. Halabi, Zaid SA[2009], "Modeling Adaptive Degraded Document Image Binarization and Optical Character System", European Journal of Scientific Research , ISSN 1450-216X Vol.28 No.1 2009, pp.14-32.

[6] Xiangyun Ye, Mohamed Cheriet, Ching Y. Suen and Ke Liu,[1999], "Extraction of bank check items by mathematical morphology", International journal on Document Analysis and Recognition, SpringerLink, Volume 2, pp 53-66.

[7] Santosh Shetty and M Shridhar[2000], "Background elimination in bank cheques using gray scale morphology", Proceedings of the Seventh International Workshop on Frontiers in Handwriting Recognition, Amsterdam, ISBN 90-76942-01-3, pp 83-92.

[8] Ferhat Fillali, Khier Benmahammed and Graini Abid[2010], "Image restoration using SVD and adaptive regularization" J. Automation \& Systems Engineering 4-3 pp. 173-181.

[9] Frank Shih[2010], "Image Processing and Mathematical Morphology Fundamentals and Applications", Wiley publications, IEEE press.

[10] Rafael C Gonzalez and Richard E Woods[2008], "Digital Image processing", Third Edition, PHI publication, 2008.

[11] Nobuyuki Otsu (1979) "A threshold selection method from gray level histograms" IEEE Trans. Systems Man and Cybernetics, Volume 9, Issue 1, 1979 pp:62-66.

[12] HIPR2, available at

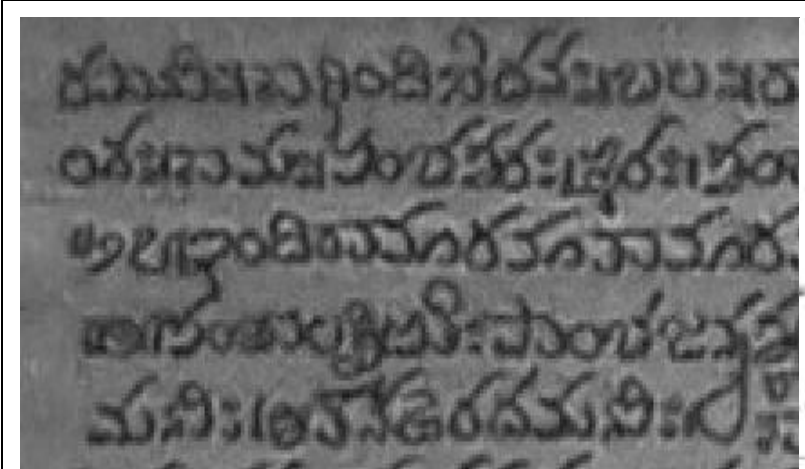

Fig 7. (a) Original Image,

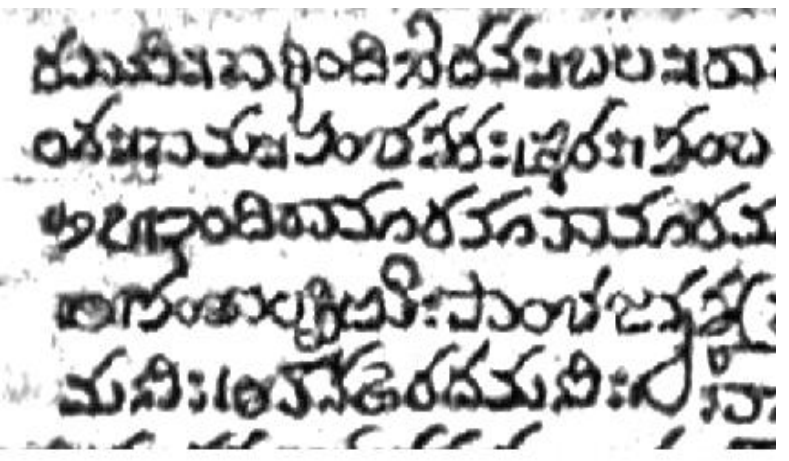

(b) Enhanced Image using proposed Method. 